\title{
Combinação dos métodos dos modos assumidos e matriz de transferência em dispositivos ópticos baseados em redes de Bragg sob deformação longitudinal
}

\author{
Paulo de Tarso Neves Junior, Carlos Alberto Bavastri, Roberson Assis Oliveira e Alexandre de \\ Almeida Prado Pohl
}

\begin{abstract}
Resumo-Este trabalho apresenta uma abordagem para simular o comportamento temporal do espectro refletido da rede de Bragg combinando dois métodos numéricos denominados modos assumidos e matriz de transferência. Essa abordagem consiste em calcular a variação espaço-temporal do campo de deslocamento e, conseqüentemente, o campo de deformação no interior da rede através do método dos modos assumidos e usar esse campo como entrada para o método da matriz de transferência calculando, finalmente, o comportamento temporal do espectro refletido da rede de Bragg. Essa abordagem é aplicada para um modulador acusto-óptico baseado em FBG e os resultados são comparados com dados experimentais relatados na literatura.
\end{abstract}

Palavras-Chave-Método dos modos assumidos, método da matriz de transferência, rede de Bragg em fibra, modulador acustoóptico.

Abstract-This work presents an approach to simulate the temporal behavior of the Bragg grating reflected spectrum combining two numerical methods named assumed modes and transfer matrix. This approach consists in calculating the timespace variation of the displacement field and, consequently, the strain field inside the grating through the assumed modes method and apply this field as input in the transfer matrix in order to calculate the time-space behavior of the Bragg grating reflected spectrum. This approach is applied in an acousto-optic modulator based on FBG and results are compared to experimental data reported in the literature.

Keywords-Assumed modes method, transfer matrix method, fiber Bragg grating, acousto-optic modulator.

\section{INTRODUÇÃO}

A proposição de modelos que permitam analisar e estimar a resposta temporal das FBGs, cuja excitação é provocada através da aplicação de tensões mecânicas longitudinais na

Carlos Alberto Bavastri, PPGEM - Programa de Pós-Graduação em Engenharia Mecânica e de Materiais.

Paulo de Tarso Neves Junior, Roberson Assis Oliveira e Alexandre de Almeida Prado Pohl, CPGEI - Programa de Pós-Graduação em Engenharia Elétrica e Informática Industrial, Universidade Tecnológica Federal do Paraná, Curitiba, Brasil. E-mails: bavastri@utfpr.edu.br, pnevesjr@gmail.com, roberson_assis@yahoo.com.br, pohl@utfpr.edu.br. fibra, pode ser de grande importância para projetar e construir dispositivos ópticos mais rápidos utilizados em telecomunicações e sensores. Para isso, recorre-se à combinação das teorias e métodos utilizados na área da mecânica e da óptica.

Há relatos sobre o uso combinado do método dos elementos finitos (MEF ou FEM - Finite Elements Method) com o Método da Matriz de Transferência a fim de simular as respostas mecânicas e ópticas, respectivamente, de um modulador acusto-óptico [1]. Uma abordagem análoga foi utilizada para simular a sensibilidade de uma FBG revestida por materiais compósitos e submetida à carga transversal estimando as deformações do espectro óptico [2] e, usando apenas o MEF sem levar em conta a deformação do espectro, para simular a sensibilidade de redes de Bragg gravadas em vários tipos de fibra sob efeito de cargas transversais e térmicas [3].

Todos os trabalhos citados acima apresentam resultados em regime permanente. Também não foi encontrado nenhum trabalho usando a combinação do método dos Modos Assumidos, analisado neste trabalho, e Matriz de Transferência para estimar o comportamento de dispositivos ópticos em regime transiente.

A escolha do método dos modos assumidos, em detrimento do método dos elementos finitos foi feita porque ao se trabalhar em uma faixa elevada de freqüências o método dos elementos finitos torna-se extremamente pesado computacionalmente; além disso, a resposta temporal já está naturalmente inclusa na formulação do método dos modos assumidos e os movimentos a serem tratados são em apenas uma dimensão, diminuindo sensivelmente o esforço computacional.

Assim como o método dos elementos finitos, a teoria do método dos modos assumidos deriva das equações de Lagrange. 


\section{METODOLOGIA}

\section{A. Equações de Lagrange}

Para sistemas mecânicos geometricamente complexos a análise dinâmica torna-se muito difícil através das relações vetoriais estabelecidas pelas Leis de Newton; por isso, soluções aproximadas podem ser encontradas usando métodos numéricos provenientes das Equações de Lagrange através de uma formulação escalar. As Equações de Lagrange têm a vantagem de desprezar completamente as forças internas do sistema e são baseadas nas relações escalares existentes entre a energia cinética, a energia potencial e o trabalho realizado pelas forças externas não conservativas.

Define-se o operador Lagrangeano como sendo

$$
L \Delta T-V
$$

onde $T$ e $V$ representam as energias cinética e potencial, respectivamente.

A transição entre o método vetorial Newtoniano e o método escalar-energético Lagrangeano é desenvolvida usando o Princípio do Trabalho Virtual, o Princípio de D'Alembert e o Princípio de Hamilton [4, 5]. Com esses três princípios chegase às equações de Lagrange em coordenadas generalizadas, $q$, que permitem derivar as equações de movimento do sistema

$$
\frac{\partial}{\partial t}\left(\frac{\partial L}{\partial \dot{q}_{j}}\right)-\frac{\partial L}{\partial q_{j}}=Q_{j}
$$

onde $Q_{j}$ são as forças de contato que atuam no sistema.

Assumindo que o sistema não sofre os efeitos giroscópico e de forças centrífugas, a energia potencial total do sistema $V$ se reduz à energia potencial dinâmica $U$.

Pode-se chegar às equações de movimento em coordenadas generalizadas na forma matricial representando as forças não conservativas generalizadas (externas e dissipativas) pela força de contato $Q_{j}$ e pelas forças de reação devido ao amortecimento viscoso de Rayleigh. Por sua vez, essas forças de reação podem ser escritas (desprezando as forças circulatórias) como

$$
\mathcal{F}=\frac{1}{2} \sum_{i=1}^{n} \sum_{j=1}^{n} c_{i j} \dot{q}_{i} \dot{q}_{j}
$$

onde $c_{i j}$ formam os componentes da matriz de amortecimento.

Dessa forma, assumindo $L=T-U$, as equações de Lagrange se reduzem a

$$
\frac{\partial}{\partial t}\left(\frac{\partial L}{\partial \dot{q}_{j}}\right)-\frac{\partial L}{\partial q_{j}}+\frac{\partial \mathcal{F}}{\partial \dot{q}_{j}}=Q_{j}
$$

A equação (4) leva a um sistema de equações diferenciais cuja forma matricial é dada por:

$$
\boldsymbol{M}\{\ddot{q}(t)\}+\boldsymbol{C}_{n \times n}\{\dot{q}(t)\}+\boldsymbol{K}_{n \times n}\{q(t)\}=\{f(t)\}
$$

onde

$\boldsymbol{M}=\left[m_{i j}\right]$ é a matriz de massa.

$\boldsymbol{C}=\left[c_{i j}\right]$ é a matriz de amortecimento viscoso.
$\boldsymbol{K}=\left[k_{i j}\right]$ é a matriz de rigidez.

$\{f(t)\}$ é o vetor de excitação em coordenadas generalizadas.

$\{q(t)\}$ é o vetor de coordenadas generalizadas.

As matrizes $\boldsymbol{M}, \boldsymbol{C}$ e $\boldsymbol{K}$ são simétricas e reais, e são fundamentais na aplicação do método dos modos assumidos.

Matematicamente a resposta do sistema é dividida em resposta transiente e resposta em regime permanente ou de estado estacionário. Para encontrar a resposta transiente do sistema é necessário obter a solução da equação homogênea de (5) fazendo $f(t)=0$. Obtendo as expressões do transiente e de regime permanente as condições iniciais do sistema são aplicadas para se obter a solução completa.

\section{B. O Método Modos Assumidos}

O método dos modos assumidos é baseado na discretização do sistema em $N$ elementos e $n$ graus de liberdade com o intuito de encontrar soluções aproximadas através de séries numéricas. Por esse método, no caso do movimento em apenas uma direção, como os movimentos longitudinais, assume-se que a solução real de um problema com condições de contorno conhecidas será encontrada através do produto de uma função espacial, convenientemente escolhida, com uma função temporal (denominada coordenada generalizada). Para o caso do movimento longitudinal em uma barra, se o objetivo for encontrar o comportamento espaço-temporal do deslocamento, a função deslocamento $u(x, t)$ será obtida resolvendo

$$
u(x, t)=\sum_{j=1}^{n} \phi_{j}(x) q_{j}(t)
$$

onde $\phi_{j}(x)$ é denominada função teste ou modos assumidos.

A equação (7) significa que a solução do problema pode ser encontrada como uma combinação linear de uma base em um espaço $n$-dimensional. Para encontrar a deformação $\varepsilon(x, t)$ basta diferenciar $u(x, t)$ em relação a $x$.

A escolha da função teste não é arbitrária; para um sistema de $n$ graus de liberdade ela deve satisfazer todas as condições geométricas e de contorno do problema e ser diferenciável em um número de vezes que seja pelo menos a metade da ordem do sistema. Como exemplo, uma função teste apropriada para o problema de uma barra engastada-livre com seção transversal não-uniforme poderia ser $\phi_{j}(x)=\operatorname{sen}\left(\frac{(2 j-1) \pi x}{2 L}\right)$.

Após a escolha de uma função teste apropriada, o próximo passo é o preenchimento das matrizes de massa, amortecimento e rigidez, além do vetor de excitação externa.

Para resolver (7) é necessário primeiramente conhecer a função de densidade $\rho(x)$, a função de área $S(x)$ e a função de massa $m(x)=\rho(x) S(x)$ ao longo da barra.

Assumindo a fibra óptica como uma barra homogênea de comprimento $L$, com funções de área e de massa conhecidas, a energia cinética $T$ será 


$$
\begin{aligned}
& T=\frac{1}{2} \int_{0}^{L} m(x)\left[\frac{\partial u(x, t)}{\partial t}\right]^{2} d x \\
& =\frac{1}{2} \sum_{i=1}^{n} \sum_{j=1}^{n} \dot{q}_{i}(t) \dot{q}_{j}(t)\left[\int_{0}^{L} m(x) \phi_{j}(x) \phi_{l}(x) d x\right]
\end{aligned}
$$

As componentes da matriz de massa serão encontradas através da expressão

$$
m_{i j}=\int_{0}^{L} m(x) \phi_{i}(x) \phi_{j}(x) d x
$$

Por sua vez, a energia potencial dinâmica será

$$
\begin{aligned}
& U=\frac{1}{2} \int_{0}^{L} E S(x)\left[\frac{\partial u(x, t)}{\partial t}\right]^{2} d x \\
& =\frac{1}{2} \sum_{i=1}^{n} \sum_{j=1}^{n} q_{i}(t) q_{j}(t)\left[E \int_{0}^{L} S(x) \frac{\partial \phi_{i}(x)}{\partial x} \frac{\partial \phi_{j}(x)}{\partial x} d x\right]
\end{aligned}
$$

onde E é módulo de Young, com os componentes da matriz de rigidez encontrados através da expressão

$$
k_{i j}=E \int_{0}^{L} S(x) \frac{\partial \phi_{i}(x)}{\partial x} \frac{\partial \phi_{j}(x)}{\partial x} d x .
$$

Assumindo um amortecimento não-proporcional distribuído de forma homogênea ao longo da barra, os coeficientes da matriz de amortecimento serão dados por

$$
c_{i j}=\int_{0}^{L} \alpha \phi_{i}(x) \phi_{j}(x) d x
$$

onde $\alpha$ é uma constante de amortecimento viscoso.

\section{O Método da Matriz de Transferência}

Para casos onde o campo de deformação varia espacialmente o espectro óptico resultante pode ser obtido através do Método da Matriz de Transferência baseado na Teoria dos Modos Acoplados [6].

Para uma rede sem gorjeio, a perturbação do índice efetivo dos modos guiados pode ser descrita como

$$
\delta n_{\text {eff }}=\delta \bar{n}_{\text {eff }}(x)\left[1+v \cos \left(\frac{2 \pi}{\Lambda}\right)\right]
$$

onde $\delta \bar{n}_{\text {eff }}$, denominado índice de modulação, é a mudança do índice de refração médio em um período da rede, $v$ é a visibilidade das franjas e $\Lambda$ o período da rede.

As soluções para as equações dos modos acoplados devido às perturbações de índice ocasionadas pela presença da rede de Bragg são encontradas através da "aproximação síncrona" relatada em $[7,8]$, resultando em

$$
\frac{d R}{d x}=i \hat{\sigma} R(x)+i \kappa S(x)
$$

$$
\frac{d S}{d x}=-i \hat{\sigma} S(x)-i \kappa^{*} R(x)
$$

onde $R$ e $S$ estão relacionados com as amplitudes dos modos propagantes e contrapropagantes, respectivamente, $\hat{\sigma}$ é o coeficiente de auto-acoplamento definido como

$$
\hat{\sigma}=\delta+\sigma
$$

sendo $\sigma$ o coeficiente de acoplamento "dc" e $\kappa$ o coeficiente de acoplamento "ac" dado por

$$
\kappa=\frac{v}{2} \sigma
$$

O termo $\delta$, por sua vez, é definido como

$$
\delta=2 \pi n_{\text {eff }}\left(\frac{1}{\lambda}-\frac{1}{\lambda_{D}}\right)
$$

onde $\lambda_{D} \equiv 2 n_{\text {eff }} \Lambda$ é o "comprimento de onda de projeto" para uma rede de Bragg com mudança de índice infinitamente pequena $\left(\delta n_{\text {eff }} \rightarrow 0\right)$ e período $\Lambda$ [9].

Dessa forma, a seguinte relação é estabelecida

$$
\begin{aligned}
& \sigma=\frac{2 \pi}{\lambda} \delta n_{e f f} \\
& \kappa=v \frac{\pi}{\lambda} \delta n_{e f f}
\end{aligned}
$$

As equações diferenciais acopladas (14) e (15) são resolvidas usando técnicas convencionais para problemas de autovalores [10]. Soluciona-se equação característica igualando o determinante a zero. O resultado, que geralmente é um polinômio, possibilita encontrar os autovalores. Posteriormente é feita a aplicação das condições de contorno para redes uniformes. Sendo um dispositivo passivo, uma rede de comprimento $L_{r}$ pode ser tratada como um quadripolo, podendo ser modelada matricialmente. Arbitrando a origem espacial no ponto de incidência da onda na rede, como mostra a Fig. 1, por conveniência normaliza-se a amplitude do campo incidente $R(0)$ de forma tal que seu valor máximo seja igual à unidade.

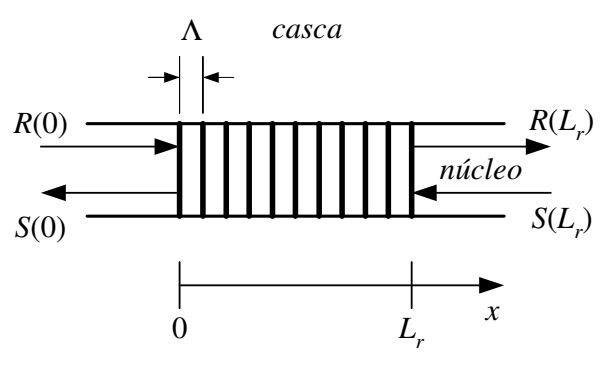

Fig. 1. Esquema da rede de Bragg como um quadripolo.

Para encontrar as condições de contorno assume-se uma onda incidente proveniente de $x=-\infty$; no início da rede a transmissão normalizada será máxima $(R(0)=1)$ e como não há reflexão além da rede, ou seja, para $x \geq L_{r}$, isso implicará 
em $S\left(L_{r}\right)=0$. Discretizando a rede em $M$ seções uniformes e definindo $R_{j}$ e $S_{j}$ como as amplitudes do campo após atravessar cada seção $j$, a propagação em cada seção é obtida através da expressão

$$
\left[\begin{array}{c}
R_{j} \\
S_{j}
\end{array}\right]=\boldsymbol{F}_{j}^{B}\left[\begin{array}{c}
R_{j-1} \\
S_{j-1}
\end{array}\right]
$$

sendo a matriz $\boldsymbol{F}_{j}{ }_{j}$ dada por

$\boldsymbol{F}_{j}^{B}=\left[\begin{array}{cc}\cosh \left(\gamma_{B} \Delta x\right)-i \frac{\hat{\sigma}}{\gamma_{B}} \sinh \left(\gamma_{B} \Delta x\right) & -i \frac{\kappa}{\gamma_{B}} \sinh \left(\gamma_{B} \Delta x\right) \\ i \frac{\kappa}{\gamma_{B}} \sinh \left(\gamma_{B} \Delta x\right) & \cosh \left(\gamma_{B} \Delta x\right)+i \frac{\hat{\sigma}}{\gamma_{B}} \sinh \left(\gamma_{B} \Delta x\right)\end{array}\right]$

onde $\Delta x$ é o comprimento da j-ésima seção e $\gamma_{B} \equiv \sqrt{\kappa^{2}-\hat{\sigma}^{2}}$.

Conhecendo as matrizes para cada seção, aplica-se as condições de contorno $\left(R(0)=1\right.$ e $\left.S\left(L_{r}\right)=0\right)$ chegando à solução final através de

$$
\left[\begin{array}{c}
R(0) \\
S(0)
\end{array}\right]=\boldsymbol{F}^{B}\left[\begin{array}{c}
R\left(L_{r}\right) \\
S\left(L_{r}\right)
\end{array}\right] \therefore\left[\begin{array}{c}
1 \\
S(0)
\end{array}\right]=\boldsymbol{F}^{B}\left[\begin{array}{c}
R\left(L_{r}\right) \\
0
\end{array}\right]
$$

sendo

$$
\boldsymbol{F}^{B}=\prod_{j=1}^{M} \boldsymbol{F}_{j}^{B} .
$$

Escrevendo $\boldsymbol{F}^{B}$ na forma

$$
\boldsymbol{F}^{B}=\left[\begin{array}{ll}
f_{11} & f_{12} \\
f_{21} & f_{22}
\end{array}\right]
$$

e substituindo em (23), resultará em

$$
\left[\begin{array}{c}
1 \\
S(0)
\end{array}\right]=\left[\begin{array}{c}
f_{11} R\left(L_{r}\right) \\
f_{21} R\left(L_{r}\right)
\end{array}\right]
$$

De (26) conclui-se que $R\left(L_{r}\right)=1 / f_{11}$. Portanto, a amplitude refletida para cada comprimento de onda pode ser encontrada através de

$$
r(\lambda)=\frac{S(0)}{R(0)}=\frac{S(0)}{1}=\frac{f_{21}}{f_{11}}
$$

sendo a potência refletida dada por $P_{r}(\lambda)=|r(\lambda)|^{2}$. Dessa forma, é possível obter a reflexão para cada comprimento de onda em um intervalo de interesse obtendo-se assim o espectro de transmissão e reflexão da rede de Bragg.

$\mathrm{O}$ deslocamento do comprimento de onda de Bragg, $\Delta \lambda_{B}$, para uma fibra submetida a uma deformação longitudinal $\varepsilon$ é dado por [11]

$$
\frac{\Delta \lambda_{B}}{\lambda_{B}}=\left(1-p_{e}\right) \varepsilon
$$

onde $\lambda_{B}$ é o comprimento de onda de Bragg para a fibra em repouso e $p_{e}$ a constante foto-elástica efetiva, que para a fibra de sílica assume o valor 0,22 .

Simula-se o efeito da variação do período da rede, causada pela deformação em seu interior, através do comprimento de onda de projeto; para isso, aplica-se (28) substituindo o comprimento de onda de Bragg pelo comprimento de onda projeto resultando em

$$
\lambda_{D}(\varepsilon(x, t))=\lambda_{D 0}\left[1+\left(1-p_{e}\right) \varepsilon(x, t)\right]
$$

onde $\lambda_{D 0}$ é uma constante representando o comprimento de onda de projeto quando a rede está em repouso. Portanto, substituindo (29) em (18), e esta em (17), obtém-se $\hat{\sigma}$ que será usado no cálculo dos elementos da matriz de transferência em cada instante de tempo.

\section{RESULTADOS}

A modulação acusto-óptica em FBGs é baseada na propagação de uma onda elástica pela rede de Bragg, produzindo uma modulação de índice de refração induzida e modificando, por deformação, o período ao longo da rede. Essa técnica é usada em lasers sintonizáveis à fibra, deslocadores de freqüência, chaves ópticas e atenuadores [1215].

Geralmente o modulador por deformação longitudinal é acionado por atuadores PZTs, excitados por um sinal harmônico com freqüências na ordem de $\mathrm{MHz}$ e sua montagem é similar à mostrada pela Fig. 2. A corneta acústica otimiza o acoplamento das ondas elásticas geradas pelo PZT para a fibra. Também é comum desgastar a casca da fibra, diminuindo o diâmetro da mesma na região da rede de Bragg a fim de se obter uma melhor interação acusto-óptica.

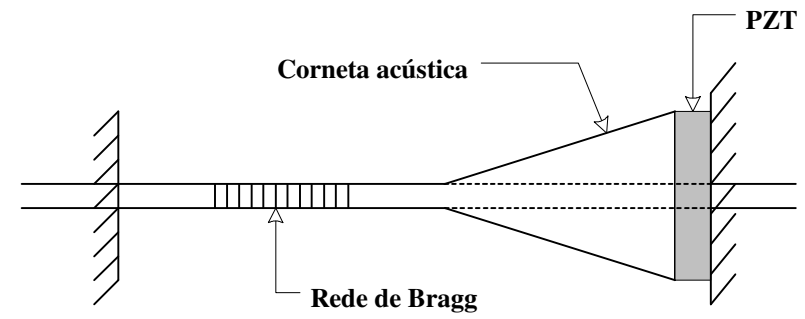

Fig. 2. Montagem típica de um modulador acusto-óptico com rede de Bragg.

A rigor o PZT deve ser modelado como uma mola ou um sistema massa-mola concentrado [16]. Porém, neste trabalho o sistema da Fig. 2 foi modelado com boa aproximação como uma barra de seção transversal não-uniforme engastada-livre com as mesmas dimensões usadas por [17] e [18]. As dimensões desse sistema são mostradas na Fig. 3. 


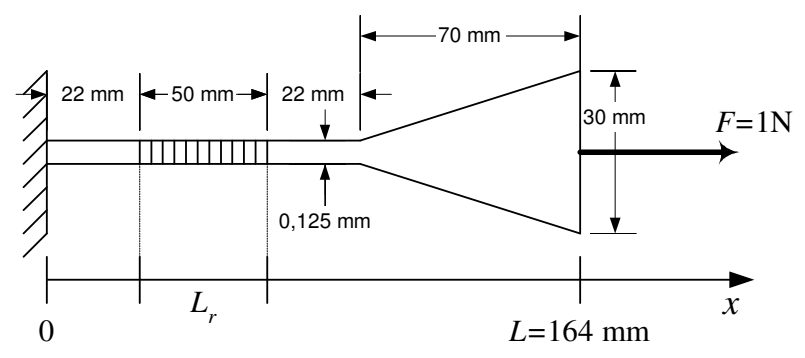

Fig. 3. Dimensões do modulador acusto-óptico [17].

Para validar a combinação dos dois métodos optou-se por usar os resultados do trabalho experimental relatado em [18]. No trabalho citado usou-se uma rede de Bragg de comprimento $L_{r}=50 \mathrm{~mm}$. As dimensões são as mesmas mostradas na Fig. 3, porém, no trabalho citado, foi feito o desgaste da casca reduzindo o diâmetro da fibra a $100 \mu \mathrm{m}$ na região da rede de Bragg. A Fig. 4 mostra o campo deformação resultante na região da rede aplicando uma força externa senoidal com amplitude de $4 \mathrm{~N}$, freqüência de $1 \mathrm{MHz}$ com uma constante de amortecimento distribuído de $\alpha=1 \mathrm{~N} . \mathrm{m} / \mathrm{s}$. Apesar dessa região ter seção transversal constante, observa-se a diminuição da amplitude do campo de deformação à medida que se aproxima a extremidade engastada (à esquerda da estrutura) e se afasta do ponto de aplicação da força (à direita da estrutura), essa diminuição é devido à dissipação de energia causada pelo amortecimento distribuído.

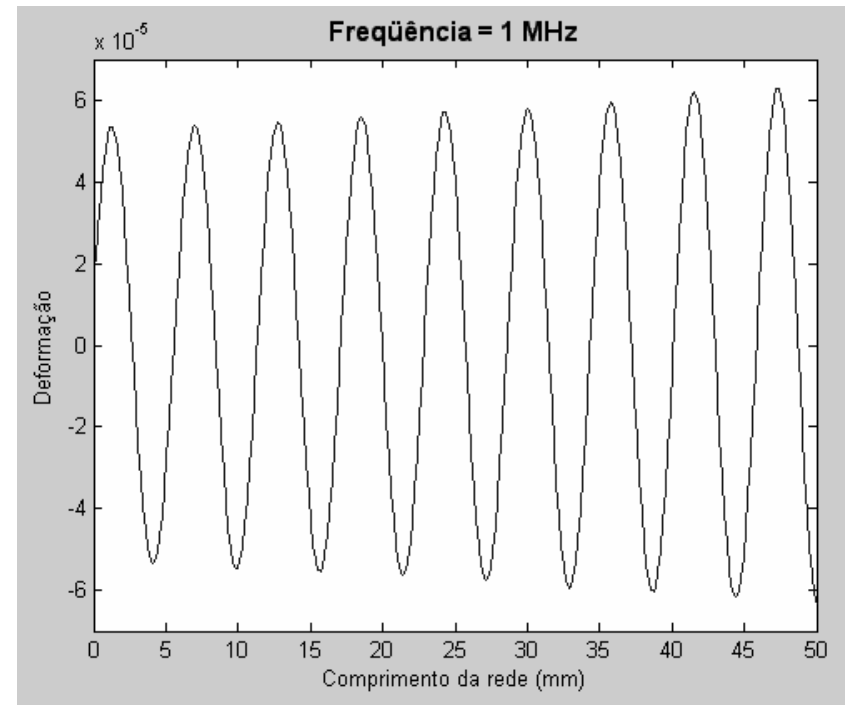

Fig. 4. Campo de deformação máxima ao longo da rede.

Não há informações sobre o índice de modulação da rede utilizada nos experimentos, porém é relatado uma estimativa para o fator $\kappa L_{r}$, o produto entre o comprimento da rede e o coeficiente de acoplamento "ac", como sendo $\kappa L_{r}=6$. Assumindo a visibilidade das franjas unitária $(v=1)$, o coeficiente de acoplamento "dc" será dado por $\sigma=2 \kappa$ (ver (17)), é possível encontrar todos os elementos da matriz de transferência.

Estimando um comprimento de onda de projeto $\lambda_{D 0}=$
1473.2 nm, o campo de deformação mostrado pela Fig. 4 foi usado como entrada no método da matriz de transferência resultando o espectro refletido mostrado pela Fig. 5-a.

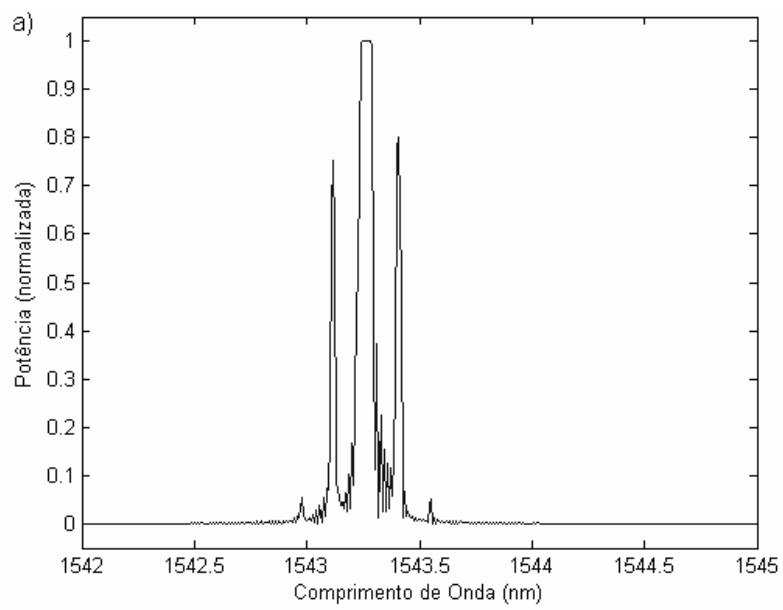

b)

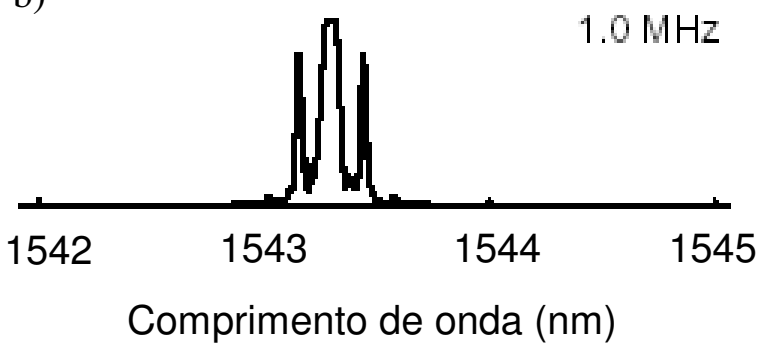

Fig. 5. a) Espectro resultante da combinação dos métodos modos assumidos e matriz de transferência. b) Espectro obtido experimentalmente em [18].

Comparando com o espectro obtido experimentalmente, mostrado na Fig. 5-b, observa-se uma grande concordância no formato, largura de banda $(60 \mathrm{pm})$ e separação entre os picos laterais de maior potência $(300 \mathrm{pm})$. Isto indica que a combinação dos dois métodos apresenta uma boa precisão. Dessa forma, é possível simular o espectro resultante variando a freqüência, as dimensões do dispositivo, o comprimento e as características da rede e até mesmo o material da corneta acústica projetando um dispositivo que atenda as especificações desejadas e minimize as possíveis limitações do atuador PZT.

A Fig. 6 mostra a deformação em regime transiente de um ponto do dispositivo localizado em $x=L / 2$ para uma constante de amortecimento distribuído $\alpha=1 \mathrm{~N} . \mathrm{s} / \mathrm{m}$. Assume-se que o regime transiente terá a mesma duração para todos os pontos da estrutura.

Nota-se que o regime estacionário acontece a partir de aproximadamente $550 \mu \mathrm{s}$. Esse tempo está de acordo com a duração do regime transiente para a emissão do laser construído com um modulador idêntico relatado em [12] e é o mesmo variando a frequiência e a força aplicada. 


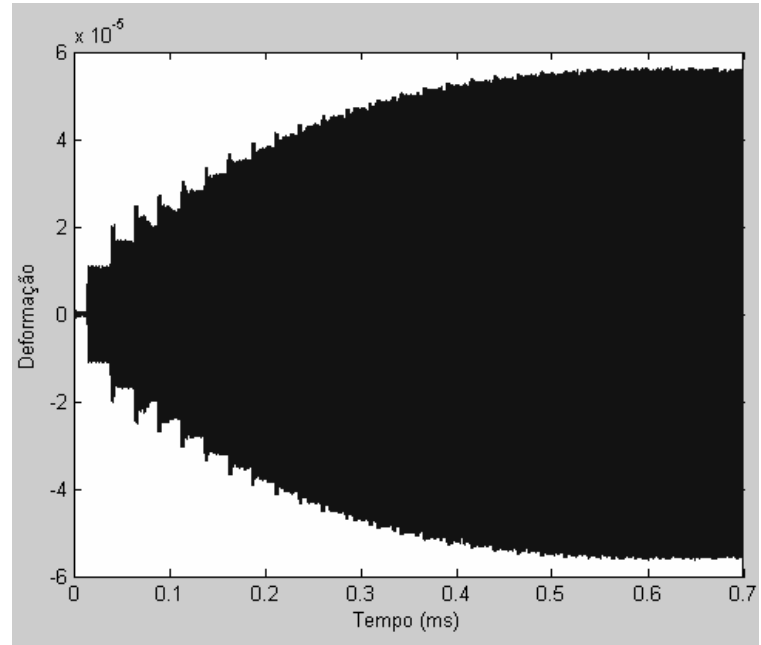

Fig. 6. Transiente da deformação para um ponto localizado em $x=L / 2 \mathrm{e}$ amortecimento $\alpha=1 \mathrm{~N} . \mathrm{s} / \mathrm{m}$.

\section{CONCLUSÕES}

O método Modos Assumidos mostrou-se eficiente na análise do comportamento temporal do comprimento de onda de Bragg em moduladores acusto-ópticos baseados em FBG submetidos à deformações unidimensionais, permitindo estimar a duração do regime transiente. Os resultados obtidos pela combinação dos métodos Modos Assumidos e Matriz de Transferência mostraram grande concordância com experimentos já realizados com um modulador acusto-óptico, podendo ser uma boa ferramenta para projetar tais dispositivos.

\section{AGRADECIMENTOS}

Paulo de Tarso Neves Junior e Roberson Assis Oliveira agradecem à Coordenação de Aperfeiçoamento de Pessoal de Nível Superior (CAPES) pelas bolsas de estudo.

\section{REFERÊNCIAS}

[1] R. A. Oliveira, P.T. Neves Jr., A. A. P. Pohl e J. T. Pereira, "Spectrum behavior of a BG-AOM under variation of design and driving parameters", IMOC 2007, pp. 716-719, 2007.

[2] M. Prabhugoud e K. Peters, "Finite element model for embedded fiber Bragg grating sensor", Smart Material and Structures, v. 15, pp. 550562, 2006.

[3] M. Prabhugoud e K. Peters, "Finite element analysis of multi-axis strain sensitivities of Bragg gratings in PM fibers", Journal of Intelligent Material Systems and Structures, v. 18, pp. 861-873, 2007.

[4] L. Meirovitch, Elements of Vibration Analysis, Singapore: McGrawHill, 1986.

[5] J. J. Espíndola, Fundamentos de Vibrações, apostila em formato de livro, UFSC, Brasil, 2000.

[6] T. Erdogan, "Fiber grating spectra", Journal of Lightwave Technology, v. 15 , n. 8, pp. 1277-1294, 1997.

[7] H. Kogelnick, Theory of optical waveguides, in Guided-Wave Optoelectronics, T. Tamir, Ed. New York: Springer-Verlag, 1990.

[8] R. Kashyap, Fiber Bragg Gratings, San Diego, CA: Academic, 1999.

[9] G. A. Miler, C. G. Askins e E. J. Friebele, "Modified F-Matrix calculation of Bragg grating spectra and its use with a novel nonlinear index growth law", Journal of Lightwave Technology, v. 24, n. 6, pp. 2416-2427, 2006.

[10] E. Kreyszig, Advanced Engineering Mathematics, New York: Wiley, 1992.
[11] K. O. Hill e G. Meltz, "Fiber Bragg grating technology fundamentals and overview", Journal of Lightwave Technology, v. 15, n. 8, pp. 12631276, 1997.

[12] M. Delgado-Pinar, D. Zalvidea, A. Diéz, J. L. Cruz e M. V. Andrés, "Wavelength-switchable fiber laser using acoustic waves", IEEE Photonics Tech. Letters, v. 17, pp. 552-554, 2005.

[13] B. Y. Kim, J. N. Blake, H. E. Engan e H. J. Shaw, "All fiber acoustooptic frequency shifter”, Optics Express, v. 11, pp. 389-391, 1986.

[14] H. S. Kim, S. H. Yun, I. K. Kwang e B. Y. Kim, "All fiber acousto-optic tunable notch filter with electronically controllable spectral profile", Optics Letters, v. 22, pp. 1476-1478, 1997.

[15] T. A. Birks, P. St. Russel e D. O. Culverhouse, "The acousto-optic effect in a single mode fiber tapers and couplers", Journal of Lightwave Technology, v. 14, pp. 2519-2529, 1996.

[16] B. M. Beadle e R. S. Weis, "Longitudinal vibration of a silica fiber segment characterized using a fiber Bragg grating", IEEE Transactions on Ultrasonics, Ferroelectrics and Frequency Control, v. 45, n. 5, pp. 1100-1104, 1998.

[17] W. F. Liu, P. St. Russel e L. Dong, "100\% efficient narrow-band acoustooptic tunable reflector using fiber Bragg grating", Journal of Lightwave Technology, v. 16, n. 11, pp. 2006-2009, 1998.

[18] M. Delgdo-Pinar, D. Zalvidea, A. Diéz, P.Péres-Millan e M. V. Andrés, "Q-switching of an all-fiber laser by acoustic modulation of a fiber Bragg grating”, Optics Express, v. 14, pp. 1106-1112, 2006. 